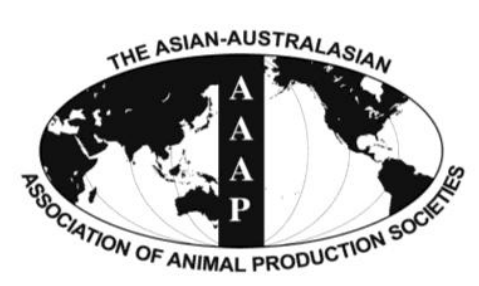

\begin{tabular}{c} 
Open Access \\
Asian Australas. J. Anim. Sci. \\
Vol. 27, No. 4 : 457-463 April 2014 \\
http://dx.doi.org/10.5713/ajas.2013.13543 \\
\hline www.ajas.info \\
pISSN 101 1-2367 elSSN 1976-5517
\end{tabular}

\title{
Genetic Analysis of Ultrasound and Carcass Measurement Traits in a Regional Hanwoo Steer Population
}

\author{
Jeong Mi Hwang, Jae Kyoung Cheong, Sam Su Kim, Bong Hwan Jung ${ }^{1}$, Myung Jae Koh ${ }^{1}$, \\ Hyeong Cheol Kim², and Yun Ho Choy ${ }^{3}$ * \\ Livestock Research Institute, National Agriculture Cooperative Federation, \\ 42-20, Daesindu-gil, Gongdo-eup, Anseong, Gyeonggi 456-824, Korea
}

\begin{abstract}
Ultrasound measurements of backfat thickness (UBF), longissimus muscle area (ULMA) and marbling score (UMS) and carcass measurements of carcass weight (CW), backfat thickness (BF), longissimus muscle area (LMA), and marbling score (MS) on 7,044 Hanwoo steers were analyzed to estimate genetic parameters. Data from Hanwoo steers that were raised, finished in Hoengseong-gun, Gangwon-do (province) and shipped to slaughter houses during the period from October 2010 to April 2013 were evaluated. Ultrasound measurements were taken at approximately three months before slaughter by an experienced operator using a B-mode real-time ultrasound device (HS-2000, FHK Co. Ltd., Tokyo, Japan) with a 3.5 MHz linear probe. Ultrasound scanning was on the left side between 13th rib and the first lumbar vertebrae. All slaughtering processes and carcass evaluations were performed in accordance with the guidelines of beef grading system of Korea. To estimate genetic parameters, multiple trait animal models were applied. Fixed effects included in the models were: the effects of farm, contemporary group effects (year-season at the time of ultrasound scanning in the models for UBF, ULMA, and UMS, and year-season at slaughter in the models for CW, BF, LMA, and MS), the effects of ultrasound technicians as class variables and the effects of the age in days at ultrasound scanning or at slaughtering as linear covariates, respectively for ultrasound and carcass measures. Heritability estimates obtained from our analyses were 0.37 for UBF, 0.13 for ULMA, 0.27 for UMS, 0.44 for CW, 0.33 for BF, 0.36 for LMA and 0.54 MS, respectively. Genetic correlations were strongly positive between corresponding traits of ultrasound and carcass measures. Genetic correlation coefficient between UBF and BF estimate was 0.938 , between ULMA and LMA was 0.767 and between UMS and MS was 0.925 . These results suggest that ultrasound measurement traits are genetically similar to carcass measurement traits. (Key Words: Hanwoo, Ultrasound, Carcass Trait, Heritability, Genetic Correlation)
\end{abstract}

\section{INTRODUCTION}

Carcass grades of finishing steers can be predicted using

\footnotetext{
* Corresponding Author: Yun Ho Choy. Tel: +82-41-580-3354, Fax: +82-41-582-1248, E-mail: ychoy000@ korea.kr

${ }^{1}$ Hoengseong Chuckhyup, 337, Hoengseong-ro, Hoengseong-eup, Hoengseong, Gangwon 225-809, Korea.

${ }^{2}$ Hanwoo Experiment Station, National Institute of Animal Science, 4937, Gyeonggang-ro, Daegwannyeong-myeon, Pyeongchang, Gangwon 232-950, Korea.

${ }^{3}$ Animal Breeding and Genetics Division, National Institute of Animal Science, 114, Sinbang 1-gil, Seobukgu, Cheonan, Chungnam 331-801, Korea.

Submitted Sept. 3, 2013; Accepted Nov. 29, 2013; Revised Jan. 20, 2014
}

ultrasound technology before they are slaughtered. In beef cattle industry, real-time ultrasound measurements on live animals may aid owners to save extra labor and feed costs. Average age at slaughter of Hanwoo steers after decision making by ultrasound prediction was decreased by 1.6 months compared to the national average age at slaughter in 2012 (Ministry of Food, Agriculture, Forestry and Fisheries, and Livestock Research Institute, NACF, 2012) .

In Korea, the National Institute of Animal Science (NIAS) and the National Agriculture Cooperative Federation (NACF) adopted ultrasound technology in late 1990s. Several researches have been focused on prediction of carcass characteristics in live Hanwoo cattle (Kim et al., 2003; Rhee et al., 2003, 2005). Application of ultrasound 
technology to genetic improvement of Hanwoo cattle has been tried by scientists as well (Lee, 2003; Roh et al., 2010). However, there were some variations in the parameters estimated by scientist depending on the time of ultrasound scanning and population of study. Kim et al. (2003) estimated correlation between ultrasound and carcass measures at about 0.60 when ultrasound scanning was applied at 17 months of age for BF, LMA, and MS, while those estimates were as high as 0.90 when ultrasound scanning was applied after 22 months of age. Song et al. (2002) reported that the correlation coefficients between ultrasound and carcass measurements: between UBF and BF, ULMA and LMA, UMS and MS were $0.75,0.57$, and 0.67 , respectively.

The objective of this study, therefore, was to evaluate phenotypic and genetic relationships between ultrasound measurements (UBF, ULMA, and UMS) and carcass measurement (CW, BF, LMA, and MS) in a regional Hanwoo steer population when ultrasound scanning procedures were applied three months before the animals were send to slaughter houses.

\section{MATERIALS AND METHODS}

\section{Data}

Hanwoo steers $(n=7,044)$, sired by 95 bulls, that were raised at 356 farms in Hoengseong-gun of Gangwon-do and that were shipped to slaughter houses from October 2010 to April 2013 were evaluated. From all datasets, carcass evaluation records and real-time ultrasound measurements with over 5 head at the same time of shipments were selected for analyses. Ultrasound measurements were recorded at approximately three months (85 days on average) before slaughter. Ultrasound measurements were taken by an experienced operator in Hoengseong-gun using a B-mode real-time ultrasound device (HS-2000, FHK Co. Ltd, Japan) with an $18 \mathrm{~cm}, 3.5 \mathrm{MHz}$ linear probe. Scanning was made by positioning the probe vertically from dorsal to ventral line parallel to the ribs between 13th thoracic vertebra and first lumbar vertebrae on the left side. Then ultrasound images were collected to the Korean cattle Synthesis System (Ver. 1.0, 2010). Collected images were analyzed by trained technicians of the National Agriculture Cooperative Federation (NACF) to estimate measures of backfat thickness (UBF), longissimus muscle area (ULMA) and marbling score (UMS). Carcass records used were cold carcass weight $(\mathrm{CW})$, backfat thickness $(\mathrm{BF})$, longissimus muscle (LMA) and marbling score (MS). Half carcasses were chilled at $0^{\circ} \mathrm{C}$ to $5^{\circ} \mathrm{C}$. After a 24-h chill, carcasses were weighted and cut between 13th rib and the first lumbar vertebrae on the left side to evaluate LMA, BF, and MS. Carcass measures of BF, LMA, and MS were assessed according to the guidelines of the beef grading system of the ministry of agriculture, food and rural affairs, Korea.

\section{Statistical analyses}

Data were first fitted to general linear models to check the significances of fixed effects on the traits under study (SAS Institute Inc., Cary, NC, 2002). For ultrasound measure traits, effect of farm, year-season at ultrasound measurements and linear covariate of age (days) at scanning were all significant sources of variation. And for carcass traits, effect of farm, year-season at slaughter, and technicians as well as age (days) at slaughter were all significant sources of variation. Effects that were accounted to have significant variation $(\mathrm{p}<0.05)$ for each dependent variable and thus were included in subsequent analyses, are presented in Table 1. Variance and covariance components were estimated with multi-trait mixed animal models using WOMBAT package (Meyer, 2007).

The animal models used in the analyses for this study were

$$
y=X \beta+Z a+e
$$

where, $\mathrm{y}$ is the vector of observations.

Table 1. Fixed effects and random effects included in the models for traits evaluated in the analyses of genetic parameters

\begin{tabular}{|c|c|c|c|c|c|}
\hline \multirow{3}{*}{ Traits $^{1}$} & \multicolumn{4}{|c|}{ Fixed effect } & \multirow{3}{*}{$\begin{array}{l}\text { Random effect } \\
\text { Additive genetic }\end{array}$} \\
\hline & \multicolumn{3}{|c|}{ Class variables } & \multirow{2}{*}{$\frac{\text { Covariate (linear) }}{\mathrm{Age}^{3}}$} & \\
\hline & Farm & Year-season $^{2}$ & Technician & & \\
\hline$\overline{\mathrm{UBF}}$ & $\mathrm{X}$ & $\mathrm{X}$ & $\mathrm{X}$ & $\mathrm{X}$ & $\mathrm{X}$ \\
\hline ULMA & $X$ & $X$ & $\mathrm{X}$ & $\mathrm{X}$ & $\mathrm{X}$ \\
\hline UMS & $X$ & $X$ & $X$ & $X$ & $X$ \\
\hline $\mathrm{CW}$ & $\mathrm{X}$ & $\mathrm{X}$ & & $\mathrm{X}$ & $\mathrm{X}$ \\
\hline $\mathrm{BF}$ & $X$ & $X$ & & $\mathrm{X}$ & $X$ \\
\hline LMA & $X$ & $X$ & & $X$ & $X$ \\
\hline MS & $\mathrm{X}$ & $\mathrm{X}$ & & $\mathrm{X}$ & $\mathrm{X}$ \\
\hline
\end{tabular}

${ }^{1}$ UBF = Ultrasound backfat thickness; ULMA = Ultrasound longissimus muscle area; UMS = Ultrasound marbling score; C = Carcass weight; $\mathrm{BF}=$ Backfat thickness; LMA = Longissimus muscle area; $\mathrm{MS}=$ Marbling score

${ }^{2}$ Year-season $=$ Year-season at ultrasound measurements or year-season at slaughter.

${ }^{3} \mathrm{Age}=$ Age (in days) at ultrasound measurements or at slaughter. 
$\beta$ is the vector of fixed effects and co-variables (farm; year-season at ultrasound measurements or year-season at slaughter; technician; age at ultrasound measurements or age at slaughter).

$\mathrm{a}$ is the vector of unknown random effects that represent the breeding value of each animal.

$\mathrm{e}$ is the vector of unknown residual random effects.

$\mathrm{X}$ and $\mathrm{Z}$ are incidence matrices that relate the records to fixed and random genetic effects.

All known pedigree relationships were traced back up to the ancestors that founded our study population. Number of animals in the pedigree identified was 19,483 animals. Inbreeding coefficient of this population on the average was $0.486 \%$.

\section{RESULTS AND DISCUSSION}

The measurements of traits from ultrasound and carcass were summarized in Table 2. The average ages at ultrasound and slaughter were 848.02 and 934.87 days, respectively. Interval between these is approximately 90 days or three months, which support the fact that ultrasound measurement was taken three months prior to slaughter. However, the intervals between these two activities were about 30 days at minimum and about 170 days at maximum. That is ultrasound measures were taken one to almost five and a half months prior to slaughter. The averages of UBF, ULMA and UMS were $10.73 \mathrm{~mm}, 88.50 \mathrm{~cm}^{2}$ and 4.36 . And the averages of CW, BF, LMA, and MS were $435.26 \mathrm{~kg}$, $13.07 \mathrm{~mm}, 90.16 \mathrm{~cm}^{2}$ and 5.68. All of the differences between corresponding measures from ultrasound and from carcass were within one SD units. Therefore, we could consider that were no extra effects on carcass traits other than animal management effects and individual animals' genetic effects that are normally accounted in genetic analysis models. The similar age structure for ultrasound measures and carcass measures were found in the studies by Cheong et al. (2012) or by Kim et al. (2012). The averages of UBF, ULMA, UMS were $9.71 \mathrm{~mm}, 86.88 \mathrm{~cm}^{2}, 4.14$, respectively in the report of Cheong et al. (2012), and 9.41 $\mathrm{mm}, 87.38 \mathrm{~cm}^{2}, 5.16$, respectively in the report of Kim et al. (2012). The averages of CW, BF, LMA, and MS were $417.94 \mathrm{~kg}, 12.91 \mathrm{~mm}, 89.92 \mathrm{~cm}^{2}, 5.31$, respectively in the report of Cheong et al. (2012), and $393.14 \mathrm{~kg}, 10.59 \mathrm{~mm}$, $89.84 \mathrm{~cm}^{2}, 5.93$, respectively in the report of Kim et al. (2012). The averages calculated in our study population were similar to those in the report of Cheong et al. (2012). The averages of carcass traits reported by Koo et al. (2011) were $415.23 \mathrm{~kg}$ for $\mathrm{CW}, 88.29 \mathrm{~cm}^{2}$ for LMA, $12.71 \mathrm{~mm}$ for $\mathrm{BF}$ and 5.42 for MS. Osawa et al. (2008) reported that the averages of CW was $445.0 \mathrm{~kg}$ and LMA, BF and MS (12 score scales) estimated at the section between the 6th and 7th thoracic vertebra were $56.8 \mathrm{~cm}^{2}, 2.3 \mathrm{~mm}$ and 5.5 at 29.6 months in Japanese Black steers. These differences might reflect regional differences in feeding steers in Korea or different methods of carcass evaluation between Korea and Japan (Japanese carcass evaluation is made between the 6th and 7 th thoracic vertebra).

Estimates of additive genetic variance for ultrasound measurements and carcass measurements by multiple trait analysis are presented in Table 3. In Korea, there have been several research works to estimate genetic parameters for carcass traits of Hanwoo steers (Yoon et al., 2002; Roh et al., 2004; Choi et al., 2006; Hwang et al., 2008). Most of the genetic (co)variances were estimated using records of steers on progeny tests at the Livestock Improvement Center. Also, the average age at slaughter of steers on progeny tests was 24 months. However, age at slaughter of steers in our study was older than that of those steers on progeny tests. Furthermore, feeding environments (feed intake, management and so on) were also different by operating farms.

Heritability estimates of the ultrasound measures were 0.43 for UBF, 0.16 for ULMA and 0.33 for UMS (Table 3). Compared to the genetic parameters estimated from this study, other reports showed some different estimates of additive genetic (co)variances ratios. Our heritability estimate for UBF was between the estimates of Roh et al. (2010). Their estimate was 0.41 when ultrasound scanning was made at 12 months of age and 0.60 when ultrasound

Table 2. Summary statistics for live animal ultrasound measurement traits and carcass traits

\begin{tabular}{|c|c|c|c|c|c|}
\hline Variables $^{1}$ & Mean & SD & $\overline{\mathrm{CV}}$ & Min & Max \\
\hline$\overline{\mathrm{UAGE}(\mathrm{d})}$ & 848.02 & 69.76 & 8.23 & 470 & 1,187 \\
\hline $\mathrm{UBF}(\mathrm{mm})$ & 10.73 & 4.20 & 39.12 & 1.25 & 40.64 \\
\hline $\operatorname{ULMA}\left(\mathrm{cm}^{2}\right)$ & 88.50 & 7.81 & 8.82 & 54.59 & 120.35 \\
\hline UMS (score 1-9) & 4.36 & 1.48 & 33.89 & 1 & 8 \\
\hline SAGE (d) & 934.87 & 70.89 & 7.58 & 637 & 1218 \\
\hline $\mathrm{CW}(\mathrm{kg})$ & 435.26 & 46.56 & 10.70 & 257 & 654 \\
\hline $\mathrm{BF}(\mathrm{mm})$ & 13.07 & 4.68 & 35.81 & 2 & 47 \\
\hline $\operatorname{LMA}\left(\mathrm{cm}^{2}\right)$ & 90.16 & 9.20 & 10.20 & 53 & 126 \\
\hline MS (score 1-9) & 5.68 & 1.77 & 31.16 & 1 & 9 \\
\hline
\end{tabular}

${ }^{1}$ UAGE = Age at ultrasound measurement; UBF = Ultrasound backfat thickness; ULMA = Ultrasound longissimus muscle area; UMS = Ultrasound marbling score; $\mathrm{SAGE}=\mathrm{Age}$ at slaughter; $\mathrm{CW}=$ Carcass weight $\mathrm{BF}=$ Backfat thickness; $\mathrm{LMA}=$ Longissimus muscle area; $\mathrm{MS}=\mathrm{Marbling}$ score . 
Table 3. Estimates of variance components and heritability for ultrasound measurements and carcass measurements from Hanwoo steers by multiple trait analysis

\begin{tabular}{lccc}
\hline Traits $^{1}$ & $\sigma_{\mathrm{A}}^{2}$ & $\sigma_{\mathrm{E}}^{2}$ & $\mathrm{~h}^{2}$ \\
\hline UBF & $6.77 \pm 1.27$ & $8.84 \pm 0.914$ & $0.43 \pm 0.07$ \\
ULMA & $7.57 \pm 1.75$ & $40.15 \pm 1.463$ & $0.16 \pm 0.04$ \\
UMS & $0.63 \pm 0.11$ & $1.25 \pm 0.081$ & $0.33 \pm 0.05$ \\
CW & $894.06 \pm 154.58$ & $909.96 \pm 109.83$ & $0.50 \pm 0.07$ \\
BF & $8.23 \pm 1.64$ & $12.62 \pm 1.19$ & $0.39 \pm 0.07$ \\
LMA & $31.85 \pm 5.80$ & $48.51 \pm 4.20$ & $0.40 \pm 0.06$ \\
MS & $1.87 \pm 0.28$ & $1.48 \pm 0.20$ & $0.56 \pm 0.07$
\end{tabular}

${ }^{1} \mathrm{UBF}=$ Ultrasound backfat thickness; ULMA = Ultrasound longissimus muscle area; UMS = Ultrasound marbling score; $\mathrm{CW}=$ Carcass weight; $\mathrm{BF}=$ Backfat thickness; LMA $=$ Longissimus muscle area; $\mathrm{MS}=$ Marbling score.

${ }^{2} \sigma_{\mathrm{A}}^{2}=$ Additive genetic variance; $\sigma_{\mathrm{E}}^{2}=$ Residual variance; $\mathrm{h}^{2}=$ Heritability.

scanning was made at 24 months of age. Stelzleni et al. (2002) reported that the heritability estimate of ultrasound fat thickness was 0.26 in yearling Brangus bulls and heifers. Kim et al. (2012) and Park et al. (2012) with repeated measures animal models reported that the heritability estimates for UBF in Hanwoo cows were 0.43 and 0.38 , respectively. In Angus steers, the heritability estimates of UBF, measured at yearling stage, was 0.39 in the report by Kemp et al. (2002), and was 0.26 in the report by MacNeil and Northcutt (2008). For ULMA, our heritability estimate was lower than those in the literature. Roh et al. (2010) reported that the estimates for ULMA were 0.57 when ultrasound images of Hanwoo steers were taken at 12 months of age and 0.57 when taken at 24 months of age. In cows, Park et al. (2012) and Kim et al. (2012) estimated heritability in Hanwoo cows with repeated measures animal models and their estimates were 0.23 and 0.31 . In the other breeds, Kemp et al. (2002) reported that the heritability estimate of ULMA in yearling Angus steers was 0.29. And Stelzleni et al. (2002) reported that the heritability estimate of ULMA was 0.31 in yearling Brangus bulls and heifers.

Our heritability estimate for UMS was similar to the estimate (0.27) of Park et al. (2012) with repeated measures animal models or the estimate (0.32) of Kim et al. (2012), both for Hanwoo cows.

Our heritability estimates for carcass traits were moderate to high: $0.50,0.39,0.40$, and 0.56 for $\mathrm{CW}, \mathrm{BF}$, LMA, and MS, respectively (Table 4).

For CW, the estimate in this study was higher than the estimates of Yoon et al. (2002), Hwang et al. (2008), or Roh et al. (2010) that ranged from 0.28 to 0.41 in Hanwoo steers slaughtered at 24 months of age after the end of progeny testing or in Japanese Black steers (Osawa et al., 2008) that were slaughtered at 25 to 37 months of age. However, the heritability estimate of CW (0.61) in Japanese Black steers and heifers slaughtered at 27 to 31 months of ages was much higher (Nogi et al., 2011) than our estimate.

The heritability estimate for LMA in this study was similar to that of Yoon et al. (2002) or that of Roh et al. (2010). However this estimate was lower than that of Hwang et al. (2008), the estimate of which was on Hanwoo steers raised in a test station and slaughtered at 24 months of age, earlier in life compared to steers observed in this study. Crew and Kemp (2001), Devitt and Wilton (2001), Kemp et al. (2002), Osawa et al. (2008), and Nogi et al. (2011) reported that the heritability estimates for LMA in their study populations were $0.54,0.45,0.44,0.43$, and 0.49 , respectively.

For BF, our estimate was similar to the estimates reported by Yoon et al (2002), or by several other researchers (Roh et al., 2004; Hwang et al., 2008) ranging from 0.39 to 0.44 in Hanwoo steers. Compared to this study, Osawa et al. (2008) estimated a lower heritability (0.22) for BF in Japanese Black steers. Crews and Kemp (2001) and Nogi et al. (2011) estimated a higher heritability, 0.55 in beef steers slaughtered at around 15 months of age and 0.51 in Japanese Black steers and heifers slaughtered at around 29 months of age. Kemp et al. (2002)'s estimate for BF in Angus steers and Devitt and Wilton (2001)'s age constant estimate in composite breed steers, both populations of which were slaughtered at about 15 months of age were 0.35 and 0.41 , respectively.

Table 4. Estimates of genetic (above the diagonal) and phenotypic (below the diagonal) correlation and their standard errors for ultrasound measurements and carcass measurements from Hanwoo steers

\begin{tabular}{lccccccc}
\hline Trait $^{1}$ & UBF & ULMA & UMS & CW & BF & LMA & MS \\
\hline UBF & & $0.638 \pm 0.107$ & $0.190 \pm 0.124$ & $0.387 \pm 0.107$ & $0.938 \pm 0.022$ & $0.139 \pm 0.131$ & $-0.039 \pm 0.119$ \\
ULMA & $0.189 \pm 0.016$ & & $0.656 \pm 0.092$ & $0.505 \pm 0.110$ & $0.637 \pm 0.109$ & $0.767 \pm 0.072$ & $0.523 \pm 0.111$ \\
UMS & $0.124 \pm 0.018$ & $0.404 \pm 0.012$ & & $0.033 \pm 0.122$ & $0.257 \pm 0.125$ & $0.625 \pm 0.086$ & $0.925 \pm 0.026$ \\
CW & $0.326 \pm 0.019$ & $0.381 \pm 0.014$ & $0.119 \pm 0.019$ & & $0.407 \pm 0.107$ & $0.378 \pm 0.106$ & $0.005 \pm 0.114$ \\
BF & $0.732 \pm 0.008$ & $0.160 \pm 0.016$ & $0.090 \pm 0.018$ & $0.356 \pm 0.018$ & & $0.181 \pm 0.132$ & $-0.002 \pm 0.123$ \\
LMA & $0.010 \pm 0.020$ & $0.460 \pm 0.012$ & $0.309 \pm 0.016$ & $0.470 \pm 0.016$ & $0.033 \pm 0.020$ & & $0.656 \pm 0.078$ \\
MS & $-0.027 \pm 0.021$ & $0.193 \pm 0.016$ & $0.619 \pm 0.011$ & $0.056 \pm 0.021$ & $-0.019 \pm 0.021$ & $0.368 \pm 0.017$ & \\
\hline
\end{tabular}

${ }^{1} \mathrm{UBF}=$ Ultrasound backfat thickness; ULMA = Ultrasound longissimus muscle area; UMS = Ultrasound marbling score; CW = Carcass weight; $\mathrm{BF}=$ Backfat thickness; LMA $=$ Longissimus muscle area; $\mathrm{MS}=$ Marbling score. 
The estimate for MS was similar to that of Crews and Kemp (2001) or that of Roh et al. (2010). Others reported somewhat lower estimates than ours that ranged from 0.44 (Hwang et al., 2008) to 0.51 (Roh et al., 2004). On the other hand, Yoon et al. (2002) reported that the heritability for MS estimated was 0.74. In Japanese Black steer population, the estimates for MS were 0.59 (Osawa et al., 2008). And in Angus steer population, the estimates were 0.42 (Kemp et al., 2002) and 0.445 (MacNeil and Northcutt, 2008). Devitt and Wilton (2001) reported a heritability estimate of 0.35 for MS from two lines of crossbred steers.

Genetic and phenotypic correlations, calculated using multiple trait animal models, among ultrasound measurements and carcass measurements are shown in Table 4. Genetic and phenotypic correlations were highly positive between UBF and BF (0.938 and 0.732), between ULMA and LMA (0.767 and 0.460) and between UMS and MS (0.925 and 0.619). A common feature in the differences in estimates between phenotypic and genetic correlation coefficients between these physiologically related traits was the fact that, in all three comparison groups, genetic correlation was always higher than phenotypic correlation. A few explanations are possible. First, lower phenotypic correlation than genetic correlation might be due to unidentified environmental effects in the models we applied in this study. Secondly, in our models, the interaction effect between individual farm's management and the situation that the animals were raised and fattened was not included. Instead, we assumed that the animals in our study population were under same environmental conditions regardless of where they were raised because all the participant farms fed animals the same concentrate diets that Hoengseong Chukhyup provided and that the fattened steers were slaughtered after the same feeding programs. Therefore, the differences between farms were unique differences in management that might be the same over the years within each farm. High genetic correlation coefficients between ultrasound and carcass measures were also found in the works of Kemp et al. (2002) or of MacNeil and Northcutt (2008), the estimates of which were in the range from 0.82 to 0.90 between $\mathrm{UBF}$ and $\mathrm{BF}$ and from 0.58 to 0.90 between ULMA and LMA.

The genetic correlation between ultrasound measurements and carcass measurements were positive except between UBF and MS. Genetic correlation coefficients between $\mathrm{UBF}$ and $\mathrm{CW}$ and between $\mathrm{BF}$ and CW were 0.387 and 0.407 , respectively. These estimates were similar to the estimates of Roh et al. (2010); 0.354 and 0.306 , respectively when ultrasound images were taken at 12 months of age; 0.456 and 0.336 , respectively when ultrasound images were taken at 24 months of age. Compared to this study, several reports (Yoon et al., 2002; Roh et al., 2004; Choi et al., 2006; Hwang et al., 2008) that dealt with Hanwoo steers on progeny tests showed lower genetic correlation estimates between $\mathrm{BF}$ and $\mathrm{CW}$, ranging from 0.08 to 0.24 .

Genetic correlation coefficients between UBF and LMA and between BF and LMA were low and positive (0.139 and 0.181 , respectively). Other reports on the genetic correlation between UBF and LMA or between BF and LMA were negative (Kemp et al., 2002; Yoon et al., 2002; Roh et al., 2004; Choi et al., 2006; Hwang et al., 2008). This may be due to the different points in time of ultrasound measurement. At around yearling age, muscle growth would be the major body compositional change while in later around the age to be slaughtered, fat deposition would be active depending on individual growth curve. Therefore, at yearling faster growing animals would build heavier muscle but thinner backfat. But at the time around slaughter, faster growing animals would build thicker backfat as well as heavier muscle in their body.

Genetic correlation coefficients between UBF and MS and between BF and MS were -0.039 and -0.002 , respectively, almost zero but slightly negative. Literature values of the genetic correlation between UBF at yearling and carcass MS at 24 months of age or between BF at yearling and carcass MS at 24 months of age in Hanwoo progeny tested steers were in the range from 0.18 to 0.24 (Yoon et al., 2002; Roh et al., 2004; Choi et al., 2006; Roh et al., 2010). Kemp et al. (2002) reported the genetic correlation estimates between UBF or BF and MS in Angus steer population were 0.29 when the traits were ageadjusted and 0.45 when the traits were weight-adjusted. Osawa et al. (2008) explained that the improvement of increasing marbling would not increase the subcutaneous fat thickness, indicating that those two traits are genetically independent. Osawa et al. (2008) reported that genetic correlations between subcutaneous fat thickness and fat area ratio for $M$. longissimusthoracis or $M$. trapezius were negative (-0.21 and -0.19$)$. Hirooka et al. (1996) reported the negative genetic correlation $(-0.12)$ between MS and subcutaneous fat thickness in Japanese Brown cattle from field records.

Genetic correlation coefficient between ULMA and CW (0.505) was higher than that between LMA and CW (0.378). The trends similar to this result were found by Roh et al. (2010) at 24 months of age. Roh et al. (2010) reported that the genetic correlation coefficients between ULMA and CW or between LMA and CW were 0.66 or 0.48 , respectively.

Genetic correlation coefficients between ULMA and MS or between LMA and MS were positive ( 0.523 or 0.656) and higher than those of the other reports. In the other reports, genetic correlation coefficients estimated between LMA of ultrasound or carcass and MS were in the range from -0.26 to 0.30 (Hirooka et al.,1996; Kemp et al., 2002; Yoon et al., 2002; Roh et al., 2004; Choi et al., 2006; 
Roh et al., 2010). Most Hanwoo breeders in the region of our study population are interested in greater genetic potentials in LMA and MS. Therefore, our positive and rather high genetic correlation between these two traits suggests that selection of the right semen and heifers with regards to these can make favorable genetic responses on both traits of their interest.

Genetic correlation coefficients between UMS and CW or between MS and CW were 0.033 or 0.005 , respectively. Genetic correlation between MS and CW was estimated to be lower than that of Yoon et al. (2002), 0.29 and Roh et al. (2004), 0.41 or that of Choi et al. (2006), 0.32. Hirooka et al. (1996) reported genetic correlation between MS and CW to be small but negative $(-0.05)$.

\section{IMPLICATIONS}

Ultrasound scanning technology applied on live animals some time before sending to slaughter houses is a very important tool to predict body compositional changes in meat animals. However, research efforts to utilize this technology as a selection tool in Hanwoo population in Korea have been limited to a few research groups. We estimated genetic parameters among ultrasound and carcass measurements and found that genetic correlations of backfat thickness, longissimus muscle area and marbling score between ultrasound and carcass measurement traits were high. Therefore, ultrasound technology can be successfully incorporated into farm management to improve meat quality of breeding animals genetically.

\section{ACKNOWLEDGEMENT}

The data for this study was provided by Hoengseong Chukhyup, Korea. We authors appreciate their efforts to collect performance data and their endeavors to improve genetic potentials of Hanwoo population in Hoengseonggun.

\section{REFERENCES}

Choi, T. J., S. D. Kim, A. J. Salces, and D. H. Baik. 2006. Genetic parameter estimation on the growth and carcass traits in Hanwoo (Korean Cattle). J. Anim. Sci. Technol. (Korean) 48:759-766.

Cheong, J. K., Y. T. Oh, H. N. Choi, C. H. Lee, K. H. Kim, K. Y. Kim, Y. H. Choy, H. C. Kim, and J. M. Hwang. 2012. Effect of geographic locations and year-seasons of birth on ultrasound scanned measures and carcass traits of Hanwoo steers. J. Anim. Sci. Technol. (Korean) 54:247-253.

Crews, D. H., Jr. and R. A. Kemp. 2001. Genetic parameters forultrasound and carcass measures of yield and quality amongreplacement and slaughter beef cattle. J. Anim. Sci. 79:3008-3020.
Devitt, C. J. B. and J. W. Wilton. 2001. Genetic correlation estimates between ultrasound measurements on yearling bulls and carcass measurements on finished steers. J. Anim. Sci. 79:2790-2797.

Hirooka, H., A. F. Groen, and M. Matsumoto. 1996. Genetic parameters for growth and carcass traits in Japanese Brown Cattle estimated from field records. J. Anim. Sci. 74:21122116.

Hwang, J. M., S. D. Kim, Y. H. Choy, H. B. Yoon, and C. J. Park. 2008. Genetic parameter estimation of carcass traits of Hanwoo steer. J. Anim. Sci. Technol. (Korean) 50:613-620.

Kemp, D. J., W. O. Herring, and C. J. Kaiser. 2002. Genetic and environmental parameters for steer ultrasound and carcass traits. J. Anim. Sci. 80:1489-1496.

Kim, H. C., S. H. Lee, C. G. Dang, G. J. Jeon, S. H. Yeon, Y. M. Cho, S. M. Lee, B. S. Yang, and J. B. Kim. 2012. Estimation of genetic parameters for ultrasound and carcass traits in Hanwoo. J. Anim. Sci. Technol. (Korean) 54:331-336.

Kim, H. C., D. H. Lee, S. B. Choi, and G. J. Jeon. 2003. Relationship between ultrasonic and carcass measures for meat qualities in Hanwoo steers. J. Anim. Sci. Technol. (Korean) 45:183-190.

Koo, Y. M., S. D. Kim, J. I. Kim, C. E. Song, K. H. Lee, Y. H. Jeoung, J. Y. Lee, H. G. Jang, B. H. Park, T. J. Choi, K. H. Cho, S. S. Lee, J. G. Lee, and H. S. Kim. 2011. Research of Statistical Model for genetic evaluation of Hanwoo carcass traits. J. Anim. Sci. Technol. (Korean) 53:283-288.

Lee, D. H. 2003. Monte Carlo simulations of selection responses for improving high meat qualities using real time ultrasound in Korean cattle. 2003. J. Anim. Sci. Technol. (Korean) 45:343354.

Ministry of Food, Agriculture, Forestry and Fisheries, and Livestock Research Institute, NACF. 2012. Report on ultrasound processing expenses support project 2012 in Korea. Nonghyup, Anseong, Korea. pp. 31-32. (in Korean).

MacNeil, M. D. and S. L. Northcutt. 2008. National cattle evaluation system for combined analysis of carcass characteristics andindicator traits recorded by using ultrasound in Angus cattle. J. Anim. Sci. 86:2518-2524.

Meyer, K. 2007. WOMBAT-A tool for mixed model analyses in quantitative genetics byrestriced maximum likelihood (REML). J. Zhejiang Univ. Sci. B 8(11):815-821.

Nogi, T., T. Honda, F. Mukai, T. Okagaki, and K. Oyama. 2011. Heritabilities and genetic correlations of fatty acid compositions in longissimus muscle lipid with carcass traits in Japanese Black cattle. J. Anim. Sci. 89:615-621.

Osawa, T., K. Kuchida, S. Hidaka, and T. Kato. 2008. Genetic parameters for image analysis traits on M. Longissimus thoracis and M. trapezius of carcass cross section in Japanese Black steers. J. Anim. Sci. 86:40-46.

Park, C. H., Y. M. Koo, B. W. Kim, D. W. Sun, J. I. Kim, C. E. Song, K. H. Lee, J. Y. Lee, Y. H. Jeoung, and J. G. Lee. 2012. Repeated Records Animal model to estimate genetic parameters of ultrasound measurement traits in Hanwoo cows. J. Anim. Sci. Technol. (Korean) 54:71-75.

Rhee, Y. J., H. K. Seok, S. J. Kim, and Y. H. Song. 2003. Early prediction of carcass yield grade by ultrasound in Hanwoo. J. Anim. Sci. Technol. (Korean) 45:327-334.

Rhee, Y. J., J. Y. Kim, S. K. Lee, and Y. H. Song. 2005. Prediction 
of carcass meat quality grade by ultrasound in Hanwoo. J. SAS. 2002. SAS/STAT User's Guide. V. 9.1. SAS Institute Inc., Anim. Sci. Technol. (Korean) 47:1095-1100. Cary, NC, USA.

Roh, S. H., B. W. Kim, H. S. Kim, H. S. Min, H. B. Yoon, D. H. Song, Y. H., S. J. Kim, and S. K. Lee. 2002. Evaluation of Lee, J. T. Jeon, and J. G. Lee. 2004. Comparison between REML and Bayesian via Gibbs sampling Algorithm with a MIX Animal Model to estimate genetic parameters for carcass traits in Hanwoo (Korean native cattle). J. Anim. Sci. Technol. (Korean) 45:719-728.

Roh, S. H., C. Y. Kim, Y. S. Won, C. J. Park, S. S. Lee, and J. G. Lee. 2010. Studies on genetic parameter estimation and sire selection to ultrasound measurement traits of Hanwoo. J. Anim. Sci. Technol. (Korean) 52:1-8. ultrasound for prediction of carcass meat yield and meat quality in Korean native cattle (Hanwoo). Asian-Aust. J. Anim. Sci. 15:591-595.

Stelzleni, A. M., T. L. Perkins, A. H. Brown Jr., F. W. Pohlman, Z. B. Johnson, and B. A. Sandelin. 2002. Genetic parameter estimates of yearling live animal ultrasonic measurements in Brangus cattle. J. Anim. Sci. 80:3150-3153.

Yoon, H. B., S. D. Kim, S. H. Na, U. M. Chang. H. K, Lee, and G. J. Jeon. 2002. Estimation of genetic parameters for carcass traits in Hanwoo steer. J. Anim. Sci. Technol. (Korean) 44:383390. 